MEDICON ENGINEERING THEMES

Volume 2 Issue 2 February 2022

Review Article

\title{
Application of Man-Machine-Environment System Engineering to Create a New World of Changqing Oilfield
}

\author{
Jiajun Sun* \\ Petrochina Changqing Oilfield Company Supervision Department, Xian, China \\ *Corresponding Author: Jiajun Sun, Petrochina Changqing Oilfield Company Supervision Department, Xian, China.
}

Received: January 27, 2022; Published: January 31, 2022

\begin{abstract}
A small oil field has been unknown in the past 30 years, why in the last 10 years it created an amazing miracle in China and even in the global oil industry? To answer this question scientifically, you have to have a scientific theory. In terms of depth and breadth of this problem, undoubtedly the most scientific theory is systems engineering theory; and from the perspective of oilfield production growth referred to on this issue, the most appropriate theory is man-machine-environment system engineering (MMESE) theory. Changqing oilfield applied MMESE to develop low-permeability reservoirs with extraordinary results, proving the value of MMESE.
\end{abstract}

Keywords: Application; MMESE; Changqing oilfield

\section{Tremendous Yield Squeezed from Low-permeability Oilfield}

Ranging across the vast northern China in the middle (N35-40, E106-111) locates an extremely significant area with unique history, and infertile earth, however, supremely abundant underground resources. To find it, it is easy to first find a twisted line in the northern China on a map of whatever size or scale, that is, Yellow River. The Yellow River flows northward and then turns to the east, and suddenly goes downwards, shaping a big Chinese character of "Ji," of which the enclosed area is just the place we are looking for. It lies in the middle of Ordos Basin, to the west of Gansu and Ningxia provinces, to the north of Inner Mongolian Plateau, to the east of Shanjin Grand Canyon, and to the south of Weihe River Plain. To give it a popular and simply name, name it Shanbei. An overall impression of Shanbei can be concluded into four colors, i.e., Red-revolution history, Black-petroleum and coal, Yellow-ecological issues, and Green-returning farmland to forestry [1]. There is a state-owned enterprise with a mission of petroleum and gas extraction on this land. Its name is Changqing Oilfield. "Speaking of oilfield, Chinese people all know Daqing Oilfield. But Changqing Oilfield is rarely known. Nevertheless, it has made a miracle surprising China and even the world" [2]. Since 2001, the new yield and reserves, two key indexes in the oil industry, of Changqing Oilfield have remained the first in China for 12 consecutive years. At least 5 million tons of petroleum and gas equivalents was increased of annual output in the recent five years, which amounts to contribute a medium-sized oilfield, like Dagang or Zhongyuan Oilfield. What is the most amazing is that Changqing Oilfield is an old oilfield which has been extracted for 43 years, and known by the world thanks to its "low pressure, low permeability, and low abundance". Particularly the low permeability, Changqing Oilfield is the most famous for this characteristic in the world. Its oil-bearing reservoir is known as the "knife grinder" without any void spaces. In the 1980s, the US energy consultation authority conducted an investigation of the Changqing Ansai Oilfield and reached a conclusion that this oilfield is a typical marginal field without any development value. Speaking of its inferior geological conditions, the core point is low permeability, which is also the key reason why the oil industry does not regard it as a promising oilfield. Layman might not understand "low permeability." Experts explain it with a metaphor that it is easy to squeeze out the oil if it is in a sponge; however, if it is in a brick, it is hard to squeeze. Comparing with the sponge, brick is low permeable. "Millidarcy"

Citation: Jiajun Sun. "Application of Man-Machine-Environment System Engineering to Create a New World of Changqing Oilfield". Medicon Engineering Themes 2.2 (2022): 24-29. 
is the basic unit for permeability in the oil industry. The smaller the value is, the lower the permeability is. Oilfield with permeability less than 50 millidarcy is regarded as the low-permeability oilfield according to the global standard, while $70 \%$ of Changqing Oilfield has permeability less than 1 millidarcy. With such a low permeability, Changqing is of course considered as an unpromising oilfield. Changqing Oilfield has always faced an awkward situation of low yield with much reservoir since 1970. The annual oil and gas output in the 1990s lingers about 1.4 million tons. In fact, Changqing Oilfield has abundant oil and gas resources. According to the general investigation of national geological resources, Ordos Basin boasts 8.588 billion tons of oil and 10.7 trillion cubic meters of gas. Changqing people have diligently persisted in squeezing out the oil and gas resources for the past 43 years. Finally, they succeeded. Year 2012 saw a yield over 45 million tons in Changqing Oilfield and 2013 will reach the target of 50 million. The western "Daqing Oilfield" is emerging and rising from the Ordos Basin with attention from the whole world. Chairman Xi Jinping, formerly the vice chairman in 2009, had a field trip to Changqing Oilfield; and in 2012 Spring Festival, Premier Wen Jiabao paid a New Year call to the workers at Changqing Oilfield.

\section{Application of MMESE in Changqing Oilfield}

Why has this unpromising Changqing Oilfield over 30 years made such tremendous wonder in the recent 10 years? To answer this question scientifically, a theory is a must. From the perspective of bread than depth, the most scientific theory is systems engineering, while considering the actual yield increase of this oilfield, it is the man-machine-environment systems engineering theory. Any oilfield can be defined as a large-scale MME system. The overall function is achieved by optimization of the three factors, namely man, machine, and environment. For a specific oilfield, the factor "man" refers to the workers with a mission to exploit the underground oil and gas. They accumulate various experiences from the practices in oil and gas exploration and development, mixed with success and failure, faults and correctness, as well as pain and pleasure. The most valuable part of these experiences becomes the operational knowledge that can be transformed into instruments, tools, machines, and equipments through technical smelting and materialization. With these devices, oil wells and gas wells are produced. All these are referred to as the factor "machine". As all the machines have their own distinct, specific and local applications and situations that constitutes the specific environment of the oilfield. Among the many environmental facets of oilfield, the most crucial factoris the property and condition of the oil and gas hidden in the thousands of meters deep underground. For Changqing Oilfield, its environmental factor is low permeability. It is extremely difficult to investigate this environmental factor hidden in the depth. Black box is the only way to understand it gradually. Fortunately, MMESE offers the technical staff a scientific method of correctly understanding the properties of hydrocarbon reservoir. There are various reasons and factors for the impressive performance of Changqing Oilfield which, never the less, can be concluded to one point, i.e., Changqing people employ machines suitable for low-permeability oilfield based on their thorough understanding of low permeability, and deliver the human operations though all kinds of ground equipments and down hole tools to the low permeable hydrocarbon reservoir, and then change this reservoir as intended. All this is MMESE applied in Changqing Oilfield. A comprehensive boundary science-man-machine-environment systems engineering-was born in China under the guidance of famous scientist Qian Xuesen in 1981. Academician Ian highly regarded this new science and wrote to HanLongshen on October 22, 1993 that you have created an important modern science in the socialist China. MMESE employs systematic scientific theory and engineering method to handle and optimize the relation of man, machine, and environment [3]. This new sciences studied human property, machine property, environmental property, man-machine relation, man-environment relation, machine-environment relation, and the overall performance of man-machine-environment system [4]. The basic theories of MMESE are control theory, model theory, and optimum theory. Control the or contributes to break the boundary between the living and non-living through some general concepts and terms, like system, information, and feedback and offers the unified Perspective and standard for researches of the three totally different and distinct objects, namely the man, machine, and environment. Model theory provides a complete set of mathematical analysis tools for the MMESE study. Evidently, MMESE requires not only qualitative analysis, but quantitative representation of the motion rules of the whole system. For this reason, proper mathematical model is introduced for different objects, and objective rules in the real world are elaborated via the mathematical language through steps of modeling, parameter identification, simulation, and verification. Optimum theory aims to seek the best or some better solutions among all the combinations of man-machine-environment systems, which is the essence of MMESE. It is a mathematical method

Citation: Jiajun Sun. "Application of Man-Machine-Environment System Engineering to Create a New World of Changqing Oilfield". Medicon Engineering Themes 2.2 (2022): 24-29. 
[5]. Although MMESE is an emerging boundary science, it has been applied widely in various industries, for example, in the exploitation of low permeability reservoir at Changqing Oilfield.

\section{How does MMESE Meet Changqing Oilfield?}

People who are familiar with MMESE may have such a question that why MMESE, as a marginal science refined from the experiences of aerospace scientists during their researches and experiments of various spacecrafts as well as with a profound national defense and military background and glorious traditions of the liberation army, was applied in the Changqing Oilfield which is only an oil exploitation enterprise on the land comparing with the aerospace researches? To answer this question, it is necessary to start from 43 years ago. Satellite Dong Fang Hong I transmitted the sound of China to the space for the first time on April 24, 1970 at Jiuquan, Gansu Province. From then, dreams of China Aerospace came true one by one, including manned space flight. 43 years have seen China become a globally recognized leading country in aerospace industry. Scientists have refined their precious experience into theories, one of which is MMESE. At the same year, on August 7, 1970, industrial oil flow is found at the No.3 well of Huachi County, Gansu Province. 49 days later, another industrial oil flow is seen at the No.1 well of Qingyang County, Gansu Province. Started from this, Changqing Oilfield has grown up from an unknown small oilfield to the No. 1 in China after 43 years. Many exploitation experiences are refined into theories. For example, academician Hu Wen who has worked in Changqing Oilfield for 34 years published the book Introduction to Low Permeability Oilfield. Both the aerospace and oil exploration course started at the same time and went through similar courses. Isn't that the fate? Moreover, people who are familiar with the history of Changqing Oilfield know that this oilfield is closely related with the liberation army. The division No. 57 of army No. 19 was totally converted into the No. 1 division of oil engineering as required by Chairman Mao. Most soldiers have participated in the construction of Changqing Oilfield. Preliminarily, this troop was led by Lanzhou Military Base delegated by the State Council and Central Military Commission. These soldiers took off the military uniform and took part in the construction of Changqing Oilfield. With such profound relation with the liberation army, oil exploration industry is closely related to the national defense and military background of MMESE. Isn't that another fate? Most oil wells and gas wells drilled in the recent ten years at Changqing Oilfield are directional wells, some are horizontal wells. Determination of direction is the core technique. It actually originated from the aerospace technology because determination of direction is also required in the aerospace industry. In addition, domestic oil industry employs the so-called HSE safety management system from this century. This system, namely an integrated management method of human health, machine safety, and environment protection, came from the Royal Dutch Shell Company. People might associate this HSE system with MMESE established by our aerospace scientists. From the inherent relation between production and safety, MMESE is the root while HSE derives from the root. Actually, MMESE over-matches HSE from the mathematization of theoretical roots, familiarity with the domestic conditions, and logical preciseness between different parts. MMESE works better than HSE at Changqing Oilfield based on the actual conditions, because MMESE is a domestic theory although it is converted from the military industry while HSE is an exotic theory that does not accord with the domestic conditions severely and requires localization. From the above analysis, that Changqing Oilfield applies MMESE consciously to solve its low permeability problem is not groundless, but natural integration due to the native conditions, profound origin, and countless connections. It is worthy to declare that MMESE is not applied in the exploitation of low permeability reservoir overnight, but following three phases. First of all, the attitude transforms from spontaneity to awareness, i.e., the concept of man-machine-environment system was established among the technicians. Secondly, the knowledge rises from perceptual to conceptual, i.e., technicians started to think about the issue systematically, taking all the three factors, man, machine, and environment into consideration. Lastly, methodology of researching the relations of the three factors changed from static to static, from doctrinal to dialectical, from one-sided to all-sided, from qualitative to quantitative, thus making the foundation for parameters discrimination and system modeling. Particularly, to point out that Changqing Oilfield, a previously unknown oilfield could strategically accept and absorb the advance technology so quickly, MMESE, from the aerospace industry had strong relations to three great minds. World famous scientist Qian Xuesen offered his enormous attention and meticulous guidance, namely his system engineering theory published in his book Technology of Organization and Management-System Engineering (Sep. 27, 1978, Wen Hui Bao, Shanghai, by Qian Xuesen, Xu Guozhi, Wang Shouyun). Although Mr. Qian had not directly focused or guided the Changqing Oilfield, his theory had been regarded as canons and read for hundreds of times by the technicians and managers. The

Citation: Jiajun Sun. "Application of Man-Machine-Environment System Engineering to Create a New World of Changqing Oilfield". Medicon Engineering Themes 2.2 (2022): 24-29. 
second great mind is Professor Long Shenzhao, who established the science of MMESE as an aerospace medicine engineering expert, held the annual MMESE symposiums for 12 consecutive years. His papers spread this discipline around the world and are learned by the technicians of Changqing Oilfield. The third leader is academician Hu Wenrui, the former general manager of Changqing Oilfield in 1999, who vigorously advocated and promoted the re-understanding of Ordos, low permeability, and ourselves. His leadership brought Changqing Oilfield out of the predicament into a bright new world. The origin of his leadership is the very MMESE.

\section{MMESE Technology to Create a New World \\ Crushing and Sand-Filling Technology to Improve Seepage of Low-permeability Reservoir}

Low-permeability reservoir features not only low output or non-output of oil well due to the ground pressure, but also constrained oil in the reservoir due to the under grown fissure and bad connectivity of stratums. To improve this situation, seepage of the reservoir needs to be changed manually, i.e., making artificial fissure by high-pressure water and fillings and into the fissure immediately to prevent crack closing, which is the so-called revolutionary crushing improvement technology. All the oil wells of Changqing Oilfield had to be crushed and transformed so as to produce oil. A series of small-scale (sand filling less than $20 \mathrm{~m}^{3}$ ), medium-scale (sand filling between 25 and $30 \mathrm{~m}^{3}$ ), and large-scale (sand filling more than $50 \mathrm{~m}^{3}$ ) tests were carried out on hundreds of wells, and eventually, the classic method was screened out. Be noted that crushing and sand filling technology is a typical MMESE, in which man is the conductor, designer, and evaluator of the crushing proposal as well as their knowledge, skills, and experiences; machine is the crushing machines, well tube, underground tools, and various auxiliary equipment; environment is the crushing liquid, crushing sand, and targeted reservoir.

\section{Pre-water-injection Technology to Increase Pressure on Low-permeability Reservoir}

When drilling the ground to the targeted stratum, oil will automatically flow into the well if the stratum has high pressure, good fluidity, and regular fissure. Then, the oil will flow to the ground with the help of pumps continuously. However, as to the low-permeability reservoir, the preliminary output fails due to the low stratum pressure and energy. Pre-water-injection technology refers to injection of water 3 or 6 months ahead of well drilling so as to maintain the original stratum pressure at 110 or $120 \%$. The ground equipment will inject water that matches the underground fluids into the stratum to increase the pressure manually. This revolutionary technology successfully solved the two global challenges in low-permeability reservoir exploration, one is low pressure and energy of the low-permeability reservoir, and the other is the continuously declining output of the low-permeability oilfield. This technology made dozens fun developed petroliferous areas into small oilfields with an annual yield over 300,000tons. The two typical examples are Jiyuan Oilfield and Xifeng Oilfield. The former had gone through five times of ups and downs without any oil. This technology made the sixth time succeed greatly. It has become a medium-sized oilfield, yielding over 3million tons in 2012. Similarly, the latter drilled out oil with the assistance of the technology at the fourth time. It made an output of 1.5 million tons in 2012. Finally, to elaborate that pre-water-injection is also a typical man-machine-environment system, in which man is the conductor, designer and evaluator of the crushing proposal as well as their knowledge, skills, and experiences; machine is the crushing machines, well tube, underground tools, and various auxiliary equipment; environment is the water source, water quality, and targeted reservoir.

\section{Cluster Well to Save Great Amount of Land}

Cluster well means that a number of oil wells are drilled with small spacing and different depth on a small area. The large cluster well is constituted with vertical wells, small-shift wells, small cluster wells, and medium cluster wells. Technologies, including drilling, investigation, crushing, testing, extracting, are transformed to support the cluster well technology, because cluster well can save great amount of land and pre-drilling costs and simplify the ground procedures. The recent twenty years have seen various cluster wells of 4,410 sets (20,250wells). Investment was saved 32million RMB and land resources saved $80,880 \mathrm{mu}\left(1 \mathrm{mu}=666.7 \mathrm{~m}^{2}\right)[6]$.

\section{Horizontal Well to Increase Oil Drainage Area}

It is undoubtedly that inclined well has more oil drainage area than that of the vertical well at the oil reservoir. How about the hori- 
zontal well? If other factors remain unchanged, the larger the oil drainage area is, the higher the output is. With the largest oil drainage area comparing with the vertical and inclined well, horizontal well can have its oil drainage area increase along with its extension in the horizontal direction; however, the area of vertical and inclined well are fixed. Horizontal well is actually a well inclined close to 90 degrees, penetrating the oil reservoir hundreds or even thousands of meters. Drilling of horizontal well is no longer an issue thanks to the perfection of directional device, direction technology and drilling equipment, plus the transformed crushing, testing, and extracting technology. The amount of horizontal wells on the Changqing Oilfield increases annually in recent three years. In 2012,21 wells are drilled and put into production at JiYuan Oilfield, each well yielding 14.3 tons per day, 8 times of that of the Aligning vertical wells. 635 wells in total are drilled on Changqing Oilfield in 2012. There are approximately 1,000 wells on the oilfield. Comparing with the vertical well, horizontal well can, with other factors remaining unchanged, output 3 times oil. It is another revolutionary technology.

\section{Ansai Mode}

Ansai Oilfield is the first low-permeability oilfield that is developed successfully and extensively in China. Eight pieces of technologies are created, namely medium scale crushing, pre-water-injection, large-scale cluster well, optimized perforation, optimized extraction, optimized well network, optimized dynamic monitoring, optimized ground procedures. Ansai Oilfield yielded more than $1 \mathrm{million}$ tons in 1997 and 3 million tons in 2008. Ground investment decreased greatly from the original 52 to $36 \%$ in the total investment.

\section{Jingan Mode}

Jianan Mode copied the Ansai Mode, plus technologies of double-tube unheated closed transportation, pressurization, regional oil transfer, oil-gas mixing transportation and ring-network water injection. The output of Jianan Oilfield surges from 1 million in 2001 to 3 million in 2007.

\section{Digital Archive Mode}

Information center selects and digitalizes all the important documents of each project under production annually, including engineering design, construction scheme, operation procedures, installation description, drawings, summaries, original records. These documents are stored in the central database for end-user logging on the server to get information. New projects or future project can obtain information from this database, so as to achieve standardized design, modular construction, digitalized management, and market-based operation. In addition, repeated working is decreased, design period shortened, labor costs saved greatly thanks to the circulation of information resources. One thing to notify is that digital archive is an information system, also atypical man-machine-environment system, in which man is the producer, manager, and user of the archive, machine is the computer and network, and environment is the effective information in the archive.

\section{Conclusions}

Development of low-permeability oilfield is not only a technical and economic issue, but also a strategic issue of energy security. Due to the increasing development of oil and gas resources, the percentage of low-permeability reservoir rises. It is discovered that the amount of low-permeability reservoir takes up $60 \%$ of the total unexploited oil reserves. It is a long-term issue confronted by China to exploit the low-permeability reservoir with high speed and amount. Changqing Oilfield applies MMESE and sets a good example for the domestic and even global oil industry in the exploitation of low-permeability reservoir.

\section{References}

1. Jiajun S. Standing on high ground in northern Shaanxi, Shaanxi Daily, the northern Shaanxi observed column (2010).

2. Yongping R and Wei W. "grindstone" take the oil "the People's Daily (2010).

3. Monograph "man-machine-environment system engineering theory and application foundation". According to Huang Ruisheng, Long sheng Science Press (2004).

4. Shengzhao L. "Man-machine-environmental systems engineering theory and its significance in the development of the produc- 
tive forces in the literature sources-machine- environment system engineering research progress". Science and Technology Press 1 (1993).

5. Chen X and Shengzhao L. The man-machine-environment systems engineering (learning) Intro J Nat 8.1 (1985).

6. Wenrui H and Zhai G. Ordos Basin oil and gas exploration and development practice and sustainable development. China Engineering (2010).

Volume 2 Issue 2 February 2022

(C) All rights are reserved by Jiajun Sun. 